УДК 539.17

\author{
V. M. Kolomietz \\ Institute for Nuclear Research, National Academy of Sciences of Ukraine, Kyiv
}

\title{
STATISTICAL DENSITY OF NUCLEAR EXCITED STATES
}

A semi-classical approximation is applied to the calculations of single-particle and statistical level densities in excited nuclei. Landau's conception of quasi-particles with the nucleon effective mass $m^{*}<m$ is used. The approach provides the correct description of the continuum contribution to the level density for realistic finite-depth potentials. It is shown that the continuum states does not affect significantly the thermodynamic calculations for sufficiently small temperatures $T \leq 1 \mathrm{MeV}$ but reduce strongly the results for the excitation energy at high temperatures. By use of standard Woods - Saxon potential and nucleon effective mass $m^{*}=0.7 m$ the $A$-dependency of the statistical level density parameter $K$ was evaluated in a good qualitative agreement with experimental data.

Keywords: level density, excited nuclei, nuclear temperature, nucleon effective mass, continuum levels.

\section{Introduction}

The methods of statistical physics and thermodynamics are essential elements of the theory of highly excited nuclei. An application of statistical methods in nuclear physics covers many observable characteristics such as the statistical level density, the resonance energies and widths, the average cross-sections of nuclear reactions, the yields in nuclear fission, etc.

A significant part in an analysis of statistical properties of excited nuclei plays the statistical level density $\rho\left(E_{e x}\right)$ for a given excitation energy $E_{\text {ex }}$ which has been a subject of many theoretical and experimental investigations in nuclear physics $[1$ 8]. A key element of a study of the statistical level density $\rho\left(E_{e x}\right)$ is the single-particle level density $g(\varepsilon)$, associated with the nuclear mean field and thereby the nuclear shell model of the noninteracting nucleons [2]. In most realistic model calculations one takes into consideration also the effects of correlated interaction, pairing effects, rotation and vibration states, etc. One can, however, expect that for the highly excited nuclei at the excitation energy $E_{e x}$ of order of the nuclear binding energy $E_{e x} \propto 7 \div 8 \mathrm{MeV}$ and higher, where the inter-level distance $\Delta E=1 \div 10 \mathrm{eV}$ is much smaller than the effect of the strong inter-particle interaction $v_{\text {int }} \gg \Delta E$, the inter-particle interaction does not perturb essentially the average level density $\rho\left(E_{e x}\right)$ leading to the inter-level mixing only. Moreover the particle-hole excitations, which are generated by a nuclear mean field and which establish the nuclear compound states at the excitation energy $E_{e x}$, represent a full set of states and the residual interaction provides a redistribution of these states only. Thus, the reasonable background for the statistical level density calculations in the case of the highly excited nuclei could be the shell-model results [8] which based on an accurate evaluation of the single-particle level density $g(\varepsilon)$. Note also that the adequate use of the realistic finite-depth potential well, such as a Woods - Saxon potential, for calculations of the statistical level density of highly excited nuclei requires the knowledge of the single-particle level density $g(\varepsilon)$ for a wide range of single-particle energy $\varepsilon$, including the continuum region [8]. A proper accounting for the continuum states is also important for determining nuclear properties in the case of the pre-equilibrium decay from states with a small exciton number $[9,10]$.

The aim of this work is the investigation of the influence of the finite depth of realistic singleparticle potential on the statistical level density $\rho\left(E_{e x}\right)$. We use the finite-depth Woods - Saxon potential and apply the semi-classical Thomas Fermi approach which is quite reasonable for the highly excited nuclei where the quantum shell effects are suppressed.

\section{Statistical level density within Fermi-gas model. Landau's conception of quasi-particles}

The statistical level density $\rho\left(E_{e x}\right) \sim \exp S\left(E_{e x}\right)$ is related to the entropy $S\left(E_{e x}\right)$ and can be evaluated by use of the Darvin - Fowler method [1,5]

$$
\rho\left(E_{e x}\right) \propto \exp S\left(E_{e x}\right)=\exp \left(2 \sqrt{a E_{e x}}\right)=\exp (2 a T),
$$

where $a$ is the level density parameter, $T$ is the temperature and the excitation energy $E_{e x} \equiv E_{e x}(T)=a T^{2}$ is referred to the ground state. In the case of simplest Fermi-gas model, the level density parameter $a$ for the nucleus with mass number $A$ can be estimated as [2]

(C) V. M. Kolomietz, 2015 


$$
a=\frac{1}{6} \pi^{2} g\left(\varepsilon_{F}\right)
$$

where $\varepsilon_{F} \approx 40 \mathrm{MeV}$ is the Fermi energy. We will also use the inverse density parameter $K$ which is derived as

$$
K=\frac{A}{a} .
$$

In two cases of the $3 D$-harmonic oscillator $(\mathrm{HO})$ and the infinite-depth square potential well (SQ) of radius $R_{0}=r_{0} A^{1 / 3}, r_{0}=1.2 \mathrm{fm}$, one has, respectively,

$$
g_{H O}\left(\varepsilon_{F}\right)=\frac{1}{4} \pi^{2} \frac{A}{\varepsilon_{F}}, a_{H O} \approx \frac{A}{16} \mathrm{MeV}^{-1}
$$

and

$$
g_{S Q}\left(\varepsilon_{F}\right)=\frac{2}{3 \pi}\left(\frac{2 m}{\hbar^{2}}\right)^{3 / 2} R_{0}^{3} \sqrt{\varepsilon_{F}}, \quad a_{S Q} \approx \frac{A}{23} \mathrm{MeV}^{-1} .
$$

Both these results for $g_{H O}\left(\varepsilon_{F}\right)$ and $g_{S Q}\left(\varepsilon_{F}\right)$ lead to a significant exceeding of the statistical level density parameter $K$, see below Fig. 5. One of cause of such kind of the exceeding for $K$ in Fig. 5 is the oversimplified assumption (2) for the level density parameter $a$ where the single-particle level density $g(\varepsilon)$ is taken at the Fermi energy $\varepsilon=\varepsilon_{F}$. Considering the heated nuclei and following the definition (1) of the statistical level density $\rho\left(E_{e x}\right)$, we should assume the high enough excitation energy $E_{e x}$ such that the temperature $T$ can be introduced. In the case of low enough temperatures $T \ll \varepsilon_{F}$ the excitation energy $E_{e x}(T)$ is derived by the calorimetric relation $E_{e x}(T) \approx a T^{2}$. This relation can be used for the thermodynamic derivation of the level density parameter $a$. Namely,

$$
a=E_{\mathrm{ex}}(T) / T^{2} .
$$

A significant point is that in the case of $T \ll \varepsilon_{F}$, the excitation energy $E_{e x}$ of a strong interacting Fermi-system is derived by the variation $\delta n(\varepsilon, T)$ of the occupation number $n(\varepsilon, T)$ in close vicinity to the Fermi energy $\varepsilon_{F}$, see below Fig. 4 . This fact allows us to apply the Landau's conception of Fermigas of quasi-particles $[11,12]$ to the strong interacting nucleons in a nucleus. The excitation energy $E_{e x}$ of the nucleus is then written as

$$
E_{e x}(T)=E(T)-E(T=0)=\int d \varepsilon \varepsilon g(\varepsilon) \delta n(\varepsilon, T),
$$

where $\delta n(\varepsilon, T)=n(\varepsilon, T)-\theta\left(\varepsilon_{F}-\varepsilon\right)$. The occupa- tion numbers $n(\varepsilon, T)$ are given by the Fermi function

$$
n(\varepsilon, T)=\frac{1}{1+\exp [(\varepsilon-\lambda) / T]},
$$

where $\lambda$ is the chemical potential which is, in general, temperature dependent $\lambda \equiv \lambda(T)$. The value of $\lambda(T)$ is obtained from the conservation of particle number $A$ :

$$
A=\int d \varepsilon g(\varepsilon) n(\varepsilon, T) .
$$

Applying the small temperature expansion with $T \ll \varepsilon_{F}$, one obtains from Eqs. (6) and (7)

$$
\lambda(T) \approx \varepsilon_{F}-\frac{\pi^{2}}{12} \frac{T^{2}}{\varepsilon_{F}} .
$$

Note also that the values of $E_{e x}(T)$ and $\lambda(T)$ are different for both the neutron and proton components. The expression (5) contains the energydependent level density $g(\varepsilon)$ and the final result for $a$ can be sensitive to the single-particle level distribution near the Fermi energy $\varepsilon_{F}$. As it was mentioned earlier, this fact is ignored in Eq. (2).

\section{Single particle level density within Thomas - Fermi (TF) and extended Thomas - Fermi (ETF) approximations}

Let us consider the number of particles $N_{\varepsilon}$ embedded in a potential well $V(\mathbf{r})$ with energy below $\varepsilon$. Using the Wigner distribution function in phase space $f(\mathbf{r}, \mathbf{p})$, one can write

$$
N_{\varepsilon}=\left.2 \int \frac{d \mathbf{r} d \mathbf{p}}{(2 \pi \hbar)^{3}} f(\mathbf{r}, \mathbf{p})\right|_{p \leq p_{\varepsilon}}
$$

where factor 2 in front of integral is caused by the spin degeneracy and $p_{\varepsilon} \equiv p_{\varepsilon}(\mathbf{r})$ is derived by the following relation

$$
\frac{p_{\varepsilon}^{2}}{2 m} \equiv \frac{p_{\varepsilon}^{2}(\mathbf{r})}{2 m}=\varepsilon-V(\mathbf{r})
$$

Note that the expression (9) must be written for both the neutron and the proton components. The singleparticle level density $g(\varepsilon)$ is derived by $N_{\varepsilon}$ as

$$
g(\varepsilon)=\frac{d}{d \varepsilon} N_{\varepsilon}
$$




\section{Thomas - Fermi approximation}

In the case of the Thomas - Fermi (TF) the distribution function $\left.f_{\mathrm{TF}}(\mathbf{r}, \mathbf{p})\right|_{p \leq p_{\mathrm{\varepsilon}}}$ is given by $\left.f_{\mathrm{TF}}(\mathbf{r}, \mathbf{p})\right|_{p \leq p_{\varepsilon}}=\Theta\left[p_{\varepsilon}(\mathbf{r})-p\right]$ and the number of particles $N_{\varepsilon}$ takes the form

$$
\begin{gathered}
N_{\mathrm{TF}, \varepsilon}=\left.2 \int \frac{d \mathbf{r} d \mathbf{p}}{(2 \pi \hbar)^{3}} f_{\mathrm{TF}}(\mathbf{r}, \mathbf{p})\right|_{p \leq p_{\varepsilon}}= \\
\quad=2 \int \frac{d \mathbf{r} d \mathbf{p}}{(2 \pi \hbar)^{3}} \Theta\left[p_{\varepsilon}(\mathbf{r})-p\right],
\end{gathered}
$$

Integrating in Eq. (12) over $\mathbf{p}$, one obtains

$$
N_{\mathrm{TF}, \varepsilon}=\frac{1}{3 \pi^{2}}\left(\frac{2 m}{\hbar^{2}}\right)^{3 / 2} \int d \mathbf{r}[\varepsilon-V(\mathbf{r})]^{3 / 2} \Theta[\varepsilon-V(\mathbf{r})] .
$$

\section{Extended Thomas - Fermi approximation}

The Thomas - Fermi approximation can be extended taking into consideration the corrections up to order of $\hbar^{2}$ in the Kirkwood's $\hbar$-expansion of the distribution function $f(\mathbf{r}, \mathbf{p})$. The corresponding extended Thomas-Fermi (ETF) distribution function $f_{\mathrm{ETF}}(\mathbf{r}, \mathbf{p})$ reads $[13,14]$

$$
\begin{gathered}
\left.f_{\mathrm{ETF}}(\mathbf{r}, \mathbf{p})\right|_{p \leq p_{\varepsilon}}=\Theta\left[p_{\varepsilon}(\mathbf{r})-p\right]+\frac{\hbar^{2}}{8 m}\left\{\left(\nabla^{2} V\right) \delta^{\prime}(h(\mathbf{r})-\varepsilon)+\right. \\
\left.+\frac{1}{3}\left[(\nabla V)^{2}+\frac{1}{m}(\mathbf{p} \cdot \nabla)^{2} V\right] \delta^{\prime \prime}(h(\mathbf{r})-\varepsilon)\right\}
\end{gathered}
$$

where $h(\mathbf{r})=p^{2} / 2 m+V(\mathbf{r})$. Substituting Eq. (14) into Eq. (9) and integrating over $\mathbf{p}$, we obtain

$$
N_{\mathrm{ETF}, \varepsilon}=\left.2 \int \frac{d \mathbf{r} d \mathbf{p}}{(2 \pi \hbar)^{3}} f_{\mathrm{ETF}}(\mathbf{r}, \mathbf{p})\right|_{p \leq p_{\varepsilon}},
$$

$$
N_{\mathrm{ETF}, \varepsilon}=N_{\mathrm{TF}, \varepsilon}-\frac{1}{24 \pi^{2}} \sqrt{\frac{2 m}{\hbar^{2}}} \int d \mathbf{r}\left[\frac{\nabla^{2} V(\mathbf{r})}{[\varepsilon-V(\mathbf{r})]^{1 / 2}}+\frac{1}{4} \frac{[\nabla V(\mathbf{r})]^{2}}{[\varepsilon-V(\mathbf{r})]^{3 / 2}}\right] \Theta[\varepsilon-V(\mathbf{r})] .
$$

Note that the value $N_{\varepsilon}$ taken at $\varepsilon=\varepsilon_{F}$ represents the total number of particles in the ground state of the Fermi system

$$
N_{\varepsilon=\varepsilon_{F}}=\left.2 \int \frac{d \mathbf{r} d \mathbf{p}}{(2 \pi \hbar)^{3}} f(\mathbf{r}, \mathbf{p})\right|_{p \leq p_{F}}=\int d \mathbf{r} \rho_{\mathrm{eq}}(\mathbf{r}),
$$

where $p_{F}$ is the Fermi momentum and $\rho_{\mathrm{eq}}(\mathbf{r})$ is the particle density in the ground state of the nucleus. Using Eqs. (16) and (17), we recover well known result for the particle density $\rho_{\mathrm{eq}, \mathrm{ETF}}(\mathbf{r})$ in the ETF approximation [15]

$$
\rho_{\mathrm{eq}, \mathrm{ETF}}(\mathbf{r})=\rho_{\mathrm{eq}, \mathrm{TF}}(\mathbf{r})-\frac{1}{24 \cdot \pi^{2}} \sqrt{\frac{2 m}{\hbar^{2}}}\left[\frac{\nabla^{2} V(\mathbf{r})}{\left[\varepsilon_{F}-V(\mathbf{r})\right]^{1 / 2}}+\frac{1}{4} \frac{[\nabla V(\mathbf{r})]^{2}}{\left[\varepsilon_{F}-V(\mathbf{r})\right]^{3 / 2}}\right] \Theta\left[\varepsilon_{F}-V(\mathbf{r})\right],
$$

where

$$
\rho_{\mathrm{eq}, \mathrm{TF}}(\mathbf{r})=\frac{1}{3 \pi^{2}}\left(\frac{2 m}{\hbar^{2}}\right)^{3 / 2}\left[\varepsilon_{F}-V(\mathbf{r})\right]^{3 / 2} \Theta\left[\varepsilon_{F}-V(\mathbf{r})\right] .
$$

The expression (16) can be simplified. Using the relation

$$
\frac{(\nabla V)^{2}}{\left[\varepsilon_{F}-V\right]^{3 / 2}}=2 \nabla \cdot \frac{\nabla V}{[\varepsilon-V]^{1 / 2}}-2 \frac{\nabla^{2} V}{[\varepsilon-V]^{1 / 2}}
$$

and the Gauss - Ostrogradsky theorem, we will reduce Eq. (16) to the following final result

$$
N_{\mathrm{ETF}, \varepsilon}=N_{\mathrm{TF}, \varepsilon}-\frac{1}{48 \pi^{2}} \sqrt{\frac{2 m}{\hbar^{2}}} \int d \mathbf{r} \frac{\nabla^{2} V(\mathbf{r})}{[\varepsilon-V(\mathbf{r})]^{1 / 2}} \Theta[\varepsilon-V(\mathbf{r})] .
$$

\section{Effective mass}

As already mentioned above, we can use the Landau's quasi-particle conception to derive the excitation energy $E_{e x}(T)$ and thereby the level density parameter $a$, see Eqs. (4) and (5). The quasiparticle conception implies that the effective mass $m^{*}<m$ of quasi-particle appears in the single particle Hamiltonian $h(r)=p^{2} / 2 m^{*}+V(\mathbf{r})$ and the mass $m$ in Eqs. (16) and (20) must be replaced by the effective mass $m^{*}$. One of consequence of such kind of replacement $m \rightarrow m^{*}$ is that the Fermi energy $\varepsilon_{F}=p_{F}^{2} / 2 m$ is shifted up

$$
\varepsilon_{F}=\frac{p_{F}^{2}}{2 m} \rightarrow \varepsilon_{F}^{*}=\frac{p_{F}^{2}}{2 m^{*}}>\frac{p_{F}^{2}}{2 m} .
$$


(Note that the Fermi-momentum $p_{F} \sim \rho_{0}^{1 / 3}$ is derived by the nuclear bulk density $\rho_{0}$ which does not depend on $m$.) The commonly used [2] parameterization of the shell model mean field $V(\mathbf{r})$ is adopted to the experimental data for the Fermi energy (or separation energy) $\varepsilon_{F}$. Therefore, the shift up of the Fermi energy $\varepsilon_{F}$ in Eq. (21) requires the relevant shift of the shell model mean field $V(\mathbf{r})$ to provide the conservation of an initially fixed separation-energy. To achieve that the bottom of the mean field $V(\mathbf{r})$ must be shifted down by factor $\mathrm{m} / \mathrm{m}^{*}$ and the mean field $V(\mathbf{r})$ is modified by the effective mass as

$$
N_{\mathrm{ETF}, \varepsilon}=\frac{1}{3 \pi^{2}} \int d \mathbf{r}\left(\frac{2 m^{*}}{\hbar^{2}}\right)^{3 / 2}\left[\varepsilon-V^{*}(r)\right]^{3 / 2} \Theta\left[\varepsilon-V^{*}(r)\right]-\frac{1}{12 \pi} \int_{0}^{\infty} d r r^{2}\left(\frac{2 m^{*}}{\hbar^{2}}\right)^{1 / 2} \frac{\Theta\left[\varepsilon-V^{*}(r)\right]}{\left[\varepsilon-V^{*}(r)\right]^{1 / 2}}\left[\frac{\partial^{2} V^{*}(r)}{\partial r^{2}}+\frac{2}{r} \frac{\partial V^{*}(r)}{\partial r}\right]
$$

Note that the nucleon effective mass $m^{*}$ is, in general, $\mathbf{r}$-dependent $m^{*} \equiv m^{*}(r)$ and includes two contributions caused by the non-locality of the nucleon-nucleon interaction (momentum-dependent effective mass $\left.m_{k}^{*}(r)\right)$ and the correlation correction (frequency dependent effective mass $\left.m_{\omega}^{*}(r)\right)[8,16]$.

For a finite depth potential $V(r)$ the singleparticle level density $g(\varepsilon)$ includes both the boundstates and the continuum-state contributions. The numerical calculations of the level density $g(\varepsilon)$

$$
V(\mathbf{r}) \rightarrow V^{*}(\mathbf{r})=\frac{m}{m^{*}} V(\mathbf{r})
$$

Finally, we obtain from Eq. (20)

$$
\begin{aligned}
N_{\mathrm{ETF}, \varepsilon} & =N_{\mathrm{TF}, \varepsilon}-\frac{1}{48 \pi^{2}} \int d \mathbf{r}\left(\frac{2 m^{*}}{\hbar^{2}}\right)^{1 / 2} \times \\
& \times \frac{\nabla^{2} V^{*}(\mathbf{r})}{\left[\varepsilon-V^{*}(\mathbf{r})\right]^{1 / 2}} \Theta\left[\varepsilon-V^{*}(\mathbf{r})\right] .
\end{aligned}
$$

Below, we will restrict ourselves to a spherical mean field $V(r)$ and rewrite Eqs. (23) and (24) as

\section{Continuum effect on the single-particle level density}

requires the high accuracy to prevent a spurious contribution to the excitation energy $E_{e x}$ of Eq. (5). Such kind of spurious contribution is caused by the free space states which are not related to the potential well $V(r)$. The corresponding free-space number of particles $N_{\text {free, } \varepsilon}$ is created by the freespace states $2 d \mathbf{r} d \mathbf{p} /(2 \pi h)^{3}$ and it is given by [8]

$$
N_{\text {free, } \varepsilon}=\frac{1}{3 \pi^{2}}\left(\frac{2 m^{*}}{\hbar^{2}}\right)^{3 / 2} \int d \mathbf{r} \varepsilon^{3 / 2} \Theta(\varepsilon)
$$

The number of states Eq. (20) should be then corrected by subtracting of the contribution $g_{\text {free }}(\varepsilon)$. The final result reads

$$
\begin{gathered}
N_{\mathrm{ETF}, \varepsilon}=\frac{4}{3 \pi} \int d r\left(\frac{2 m^{*}}{\hbar^{2}}\right)^{3 / 2} r^{2}\left\{\left[\varepsilon-V^{*}(r)\right]^{3 / 2} \Theta\left[\varepsilon-V^{*}(r)\right]-\varepsilon^{3 / 2} \Theta(\varepsilon)\right\}- \\
-\frac{1}{12 \pi} \int_{0}^{\infty} d r\left(\frac{2 m^{*}}{\hbar^{2}}\right)^{1 / 2} r^{2} \frac{\Theta\left[\varepsilon-V^{*}(r)\right]}{\left[\varepsilon-V^{*}(r)\right]^{1 / 2}}\left[\frac{\partial^{2}}{\partial r^{2}} V^{*}(r)+\frac{2}{r} \frac{\partial}{\partial r} V^{*}(r)\right] .
\end{gathered}
$$

Note that the procedure of subtracting of the freestate contribution $N_{\text {free, } \varepsilon}$ from the continuum states agrees with the results of phase-shift analysis of the single-particle levels in continuum (resonance states), see Refs. [8, 17]. In particular, the subtraction of the free-state contribution $N_{\text {free, } \varepsilon}$ provides the fulfillment of the Levinson's theorem [18] which establishes the relation between the phase shift at zero energy and the number of bound states.

\section{Single particle level density}

Evaluating the single particle density $g(\varepsilon)$, we will apply our consideration to two potentials $V(r)$ : (i) Trapezoidal (TR) potential well $V_{\mathrm{TR}}(r)$ and (ii) Woods - Saxon (WS) potential $V_{\mathrm{wS}}(r)$.

\section{Trapezoidal potential well}

We will adopt the following form of trapezoidal mean field 


$$
V_{\mathrm{TR}}(r)=\left\{\begin{array}{cc}
V_{0} & r<R-D \\
(1 / 2) V_{0}[1-(r-R) / D] & R-D \leq r \leq R+D, \\
0 & r>R+D
\end{array}\right.
$$

where

$$
\begin{gathered}
V_{0}=-54+33 t_{3} \frac{N-Z}{A} \mathrm{MeV} \\
R=\frac{R_{0}}{\left[1+(D / R)^{2}\right]^{1 / 3}}, R_{0}=1.12 \cdot A^{1 / 3}+1.0 \mathrm{fm}, \\
D=\pi d, d=0.7 \mathrm{fm} .
\end{gathered}
$$

Here, $t_{3}=1$ for a neutron and -1 for a proton. Using the trapezoidal potential (27) and the Thomas Fermi approximation, the single particle density $g_{\mathrm{TF}}(\varepsilon)$ can be evaluated analytically. The final result reads, see also Ref. [8],

$g_{\mathrm{TF}}(\varepsilon)=\frac{1}{2 \pi^{2}}\left(\frac{2 m^{*}}{\hbar^{2}}\right)^{3 / 2}\left(\varepsilon-V_{0}^{*}\right)^{1 / 2}\left[1+2 x+\frac{8}{5} x^{2}+\frac{16}{35} x^{3}\right]$,

where

$$
x=-\frac{2 D\left(\varepsilon-V_{0}^{*}\right)}{(R-D) V_{0}^{*}} .
$$

The free-gas level density $g_{\text {free }}(\varepsilon)$ is derived by Eqs. (25) and (11)

$$
\begin{gathered}
g_{\text {free }}(\varepsilon)=\frac{1}{2 \pi^{2}}\left(\frac{2 m^{*}}{\hbar^{2}}\right)^{3 / 2} \times \\
\times \frac{4 \pi(R+D)^{3}}{3} \varepsilon^{1 / 2}\left[1+2 y+\frac{8}{5} y^{2}+\frac{16}{35} y^{3}\right],
\end{gathered}
$$

where

$$
y=-\frac{2 D \varepsilon}{(R+D) V_{0}^{*}} .
$$

In Fig. 1 we show the neutron single-particle level density for the nucleus ${ }^{208} \mathrm{~Pb}$ obtained from Eqs. (28) and (29). The dashed lines are obtained using equations (28) and (29) for the infinite trapezoidal potential, and the solid lines are for the finite-depth trapezoidal potential of Eq. (27). The lines 1 and 2 are for different values of diffuseness parameter $d=0.7 \mathrm{fm}$ and $d=0.1 \mathrm{fm}$.

As one can see from Fig. 1, the subtraction of the continuum states reduces significantly the single-particle level density for $\varepsilon>0$. Note also that, due to the finite size of the nuclear potential well, one has for the continuum region $\varepsilon>0$ that $g(\varepsilon)$ decreases with increasing $\varepsilon$. That means that

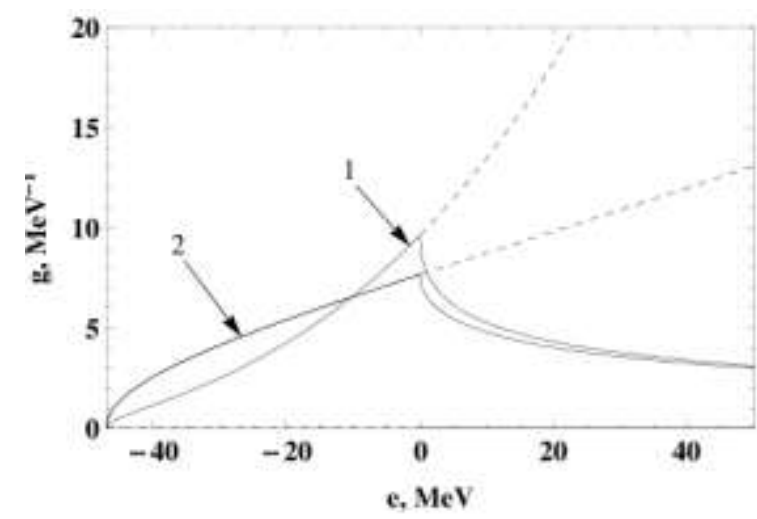

Fig. 1. The neutron single-particle level density for nucleus ${ }^{208} \mathrm{~Pb}$ for the trapezoidal potential assuming $m^{*}=m$. The lines 1 and 2 are for different values of diffuseness parameter $d=0.7 \mathrm{fm}$ and $d=0.1 \mathrm{fm}$. The dashed lines are for the infinite trapezoidal well.

a proper treatment of the continuum is important for determining nuclear properties such as the level density of hot nuclei and the exciton level density at high excitation energy. The results of Fig. 1 show also that the reduction of the diffuseness parameter $d$ of surface layer changes the behavior of level density $g(\varepsilon)$, which is $g(\varepsilon) \sim \varepsilon^{2}$ (oscillator-like) for a smooth surface (curve 1 in Fig. 1) to $g(\varepsilon) \sim \sqrt{\varepsilon}$ (square well-like) for a sharp surface (curve 2 in Fig. 1).

The $\hbar^{2}$-corrections and the corresponding ETF values of the level density $g(\varepsilon)$ and the number of particles $N_{\varepsilon}$ of can be evaluated using Eqs. (11), (26) and (27). For the $\hbar^{2}$-correction $N_{\text {corr, } \varepsilon}$ to the number of particles one obtains

$$
N_{\text {corr }, \varepsilon}=\frac{V_{0}^{*}}{12 \pi D}\left(\frac{2 m^{*}}{\hbar^{2}}\right)^{1 / 2} \int_{R-D}^{R+D} d r r \frac{\Theta\left[\varepsilon-V_{1}^{*}(r)\right]}{\left[\varepsilon-V_{1}^{*}(r)\right]^{1 / 2}},
$$

where $V_{1}^{*}(r)=V_{0}^{*}[1-(r-R) / D] / 2$.

In Fig. 2 we have plotted the number of particles $N_{\varepsilon}$ embedded in trapezoidal potential with energy below $\varepsilon$ evaluated within the Thomas-Fermi approximation in comparison with the $\hbar^{2}$-correction $N_{\text {corr, } \varepsilon}$ which is provided by the ETF approximation of Eq. (30). As one can see the $\hbar^{2}$-correction $N_{\text {corr, } \varepsilon}$ is negative and negligible small, i.e., the corrections due to the ETF approximation play the minor role. Following this result, we will below restrict ourselves to the Thomas - Fermi approximation only. 


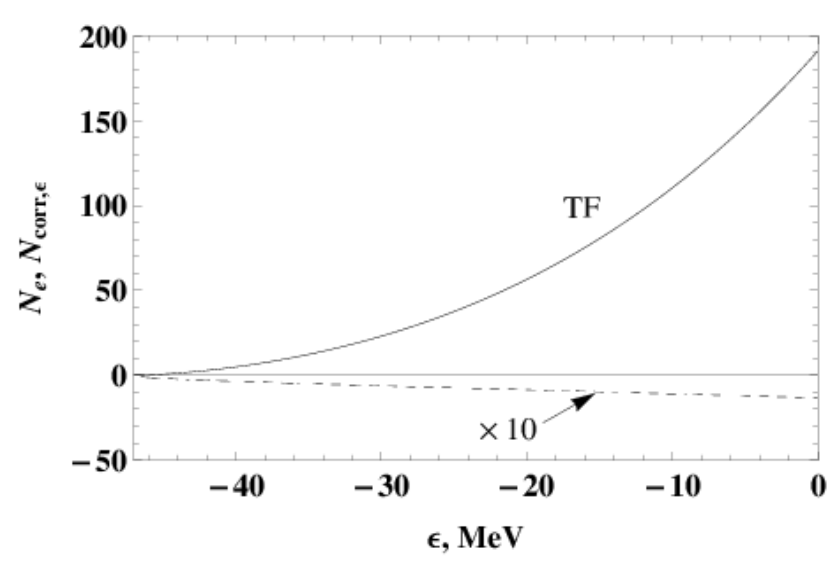

Fig. 2. Number of particles $N_{\varepsilon}$ within Thomas - Fermi approximation (solid line) and $\hbar^{2}$-correction $N_{\text {corr }, \varepsilon}$ (dashed line) for the trapezoidal potential for the nucleus ${ }^{208} \mathrm{~Pb}$ with $m^{*}=m$.

\section{Woods - Saxon potential}

We will use the Woods - Saxon potential in the following form

$$
V_{\mathrm{wS}}(r)=\left[V_{0}+33 t_{3} \frac{N-Z}{A}\right] \frac{1}{1+\exp \left[\left(r-R_{0}\right) / d\right]} .
$$

We will adopt the following parameters $V_{0}=-58 \mathrm{MeV}, \quad R_{0}=r_{0} A^{1 / 3}, \quad r_{0}=1.12 \mathrm{fm} \quad$ and $d=0.5 \mathrm{fm}$ [2]. The mean field of protons includes also the Coulomb potential

$$
V_{C}= \begin{cases}\frac{Z e^{2}}{R_{C}} \frac{1}{2}\left[3-\left(\frac{r}{R_{C}}\right)^{2}\right] & \left(r \leq R_{C}\right) \\ \frac{Z e^{2}}{r} & \left(r \geq R_{C}\right),\end{cases}
$$

where $\quad e^{2}=1.44 \mathrm{MeV} \cdot \mathrm{fm}, \quad R_{C}=r_{C} A^{1 / 3} \quad$ and $r_{C}=1.24 \mathrm{fm}$.

Using the results of previous sections, we have evaluated the single-particle level density for the WS potential. Fig. 3 shows the TF result for the neutron level density $g_{n}(\varepsilon)$ for the nucleus ${ }^{208} \mathrm{~Pb}$ with effective mass $m^{*}=m$ (dashed line) and $m^{*}=0.8 m$ (solid line).

Note that the effective mass $m^{*} \neq m$ affects significantly the slope of curve $g_{n}(\varepsilon)$ for the bound states with energy $e<0$. This fact plies an important role for the calculations of the excitation energy and the entropy of the heated nuclei, see next Section.

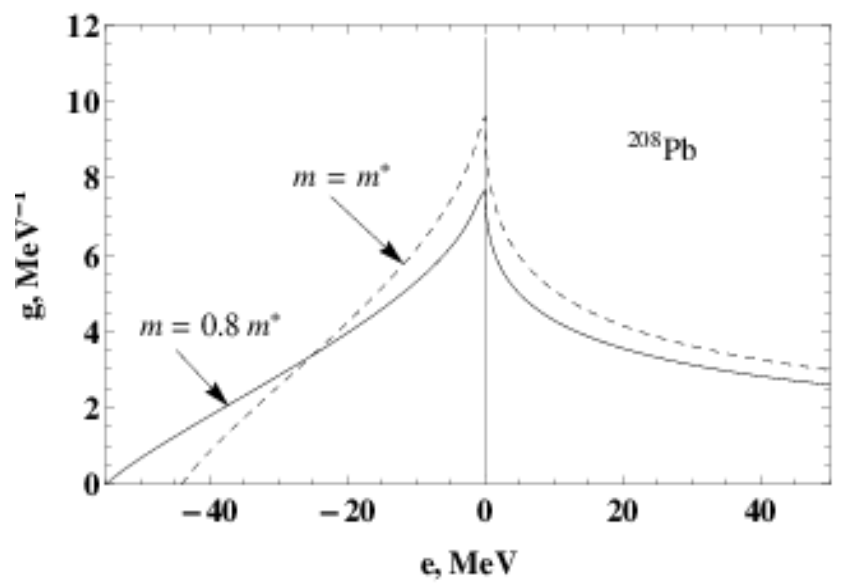

Fig. 3. The neutron level density $g_{n}(\varepsilon)$ for the nucleus ${ }^{208} \mathrm{~Pb}$ within the Thomas - Fermi approximation. The dashed line is for $m^{*}=m$ and the solid line is for $m^{*}=0.8 m$.

\section{Nuclear excitation energy and statistical level density parameter}

Assuming the Landau's conception of Fermi-gas of quasiparticles, we will apply Eq. (5) to evaluate the nuclear excitation energy $E_{e x}$. The Landau's conception of quasiparticles requires that the integrand $f(\varepsilon)=\varepsilon g(\varepsilon) \delta n(\varepsilon, T)$ in Eq. (5) should be localized near the Fermi energy $\varepsilon_{F}$. In Fig. 4 we have plotted the integrand $f(\varepsilon)$ for the nucleus ${ }^{208} \mathrm{~Pb}$ for two temperatures $T=1 \mathrm{MeV}$ and $T=3 \mathrm{MeV}$.

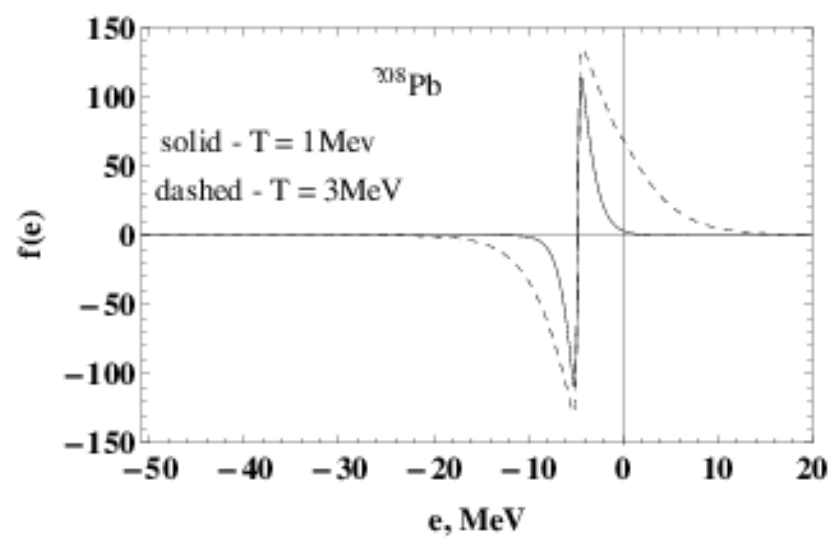

Fig. 4. The integrand $f(\varepsilon)=\varepsilon g_{n}(\varepsilon) \delta n(\varepsilon, T)$ in Eq. (5) for the neutron excitation energy $E_{e x}$ for the nucleus ${ }^{208} \mathrm{~Pb}$ for two temperatures $T=1 \mathrm{MeV}$ and $T=3 \mathrm{MeV}$.

As can be seen from Fig. 4, the integrand $f(\varepsilon)$ of Eq. (5) is localized near the Fermi energy ( $\varepsilon_{F}=-8.37 \mathrm{MeV}$ for ${ }^{208} \mathrm{~Pb}$ in Fig. 4) quite well even for the relatively high temperature $T=3 \mathrm{MeV}$. This fact confirms the Landau's conception of quasiparticles and allows one to use Eq. (5) to 
evaluate the nuclear excitation energy. Note also that for high temperatures (e.g., $T=3 \mathrm{MeV}$ in Fig. 4) the integrand $f(\varepsilon)$ in Eq. (5) penetrates into the region of continuum states $e>0$ and the continuum effect on the single-particle level density (see previous Section) has to be taken into consideration.

In Table we show the results of calculations of the Fermi energy $\varepsilon_{F, q}$, the level density parameters $a_{q} \quad(q=n$ for neutron and $q=p$ for proton) and the inverse parameter $K=A /\left(a_{n}+a_{p}\right)$ for three spherical nuclei ${ }^{40} \mathrm{Ca},{ }^{48} \mathrm{Ca}$ and ${ }^{208} \mathrm{~Pb}$. All results were obtained wthin the Thomas - Fermi approximation: TF with $m^{*}=m$ and $\mathrm{TF}^{*}$ with $m^{*}=0.7 m$.

The results of calculation of the Fermi energy $\varepsilon_{F, q}$, the statistical density parameter $a_{q}$ and the inverse density parameter $K$ for different nuclei. The calculations were performed within the Thomas - Fermi (TF) approximation with Woods - Saxon potential

\begin{tabular}{|l|l|l|l|l|l|l|l|l|l|l|}
\hline \multirow{2}{*}{ Nuclei } & $\begin{array}{c}\varepsilon_{F, n} \\
\mathrm{TF}^{*}\end{array}$ & $\begin{array}{c}\varepsilon_{F, p} \\
\mathrm{TF}^{*}\end{array}$ & $\begin{array}{c}a_{n} \\
\mathrm{TF}^{*}\end{array}$ & $\begin{array}{c}a_{p} \\
\mathrm{TF}^{*}\end{array}$ & $\begin{array}{c}K \\
\mathrm{TF}^{*}\end{array}$ & $\begin{array}{c}\varepsilon_{F, n} \\
\mathrm{TF}\end{array}$ & $\begin{array}{c}\varepsilon_{F, p} \\
\mathrm{TF}\end{array}$ & $\begin{array}{c}a_{n} \\
\mathrm{TF}\end{array}$ & $\begin{array}{c}a_{p} \\
\mathrm{TF}\end{array}$ & $\begin{array}{c}K \\
\mathrm{TF}\end{array}$ \\
\hline${ }^{40} \mathrm{Ca}$ & -17.25 & -5.49 & 3.04 & 3.03 & 6.59 & -12.08 & -3.85 & 4.36 & 3.40 & 5.15 \\
\hline${ }^{48} \mathrm{Ca}$ & -7.68 & -17.62 & 5.14 & 2.77 & 6.07 & -5.38 & -12.34 & 7.05 & 3.96 & 4.36 \\
\hline${ }^{208} \mathrm{~Pb}$ & -6.95 & -8.39 & 5.92 & 8.74 & 8.43 & -4.87 & -5.88 & 20.78 & 11.99 & 6.34 \\
\hline
\end{tabular}

* Means that the effective mass $m^{*}=0.7 m$ was used.

Fig. 5 shows the comparison of the experimental data (solid points) and the evaluated values of inverse parameter $K$ within the TF approximation with Woods - Saxon potential.

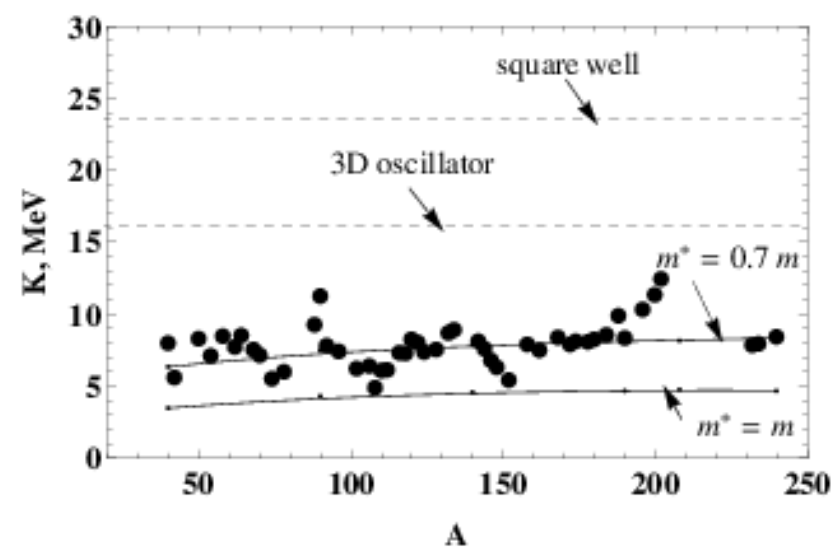

Fig. 5. Experimental values of $K$ for even-even nuclei (solid points) from Ref. [5]. The solid lines are for the TF calculations with Woods - Saxon potential in two cases $m^{*}=m$ and $m^{*}=0.7 m$. The dashed lines are for the 3D oscillator potential and the infinite-depth square potential well, see above $g_{H O}\left(\varepsilon_{F}\right)$ and $g_{S Q}\left(\varepsilon_{F}\right)$.

The results of Fig. 5 demonstrate a quite satisfactory description of average behavior of the $A$-dependency of the statistical level-density parameter $K$ obtained within the Thomas - Fermi approximation by use of the realistic Woods - Saxon potential. Two aspects are important for such kind of description: (i) the energy dependency of single particle level density $g(\varepsilon)$, in particular, the slope of curve $g(\varepsilon)$ in vicinity of the Fermi energy $\varepsilon \approx \varepsilon_{F}$; (ii) the effective mass of nucleon $m_{q}^{*}<m_{q}$ which reflects the Landau's conception of the Fermi gas of quasiparticles.

The continuum states in single-particle level density $g(\varepsilon)$ do not affect significantly the thermodynamic calculations of the nuclear excitation energy $E_{e x}$ and the statistical level density parameter $K$ for sufficiently small temperatures $T \leq 1 \mathrm{MeV}$. However, the procedure of the correct subtracting of the free-state contribution from the continuum states plies an important role for high temperatures. The influence of the continuum correction to the single-particle level density on the nuclear caloric curve $E_{e x}(T)$ is shown in Fig. 6.

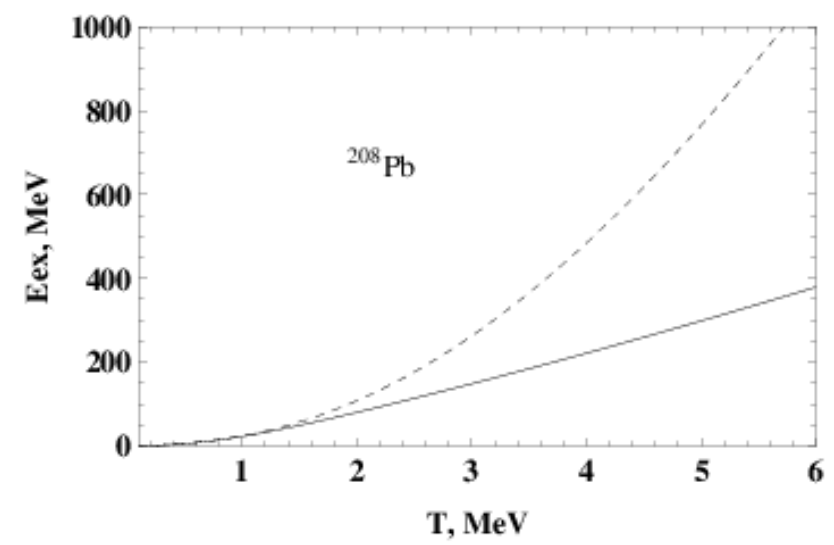

Fig. 6. The caloric curve of nucleus ${ }^{208} \mathrm{~Pb}$. The dashed line is without of subtracting of the free-state contribution $N_{\text {free, } \varepsilon}$ (see Eq. (25)) from the continuum states and the solid line is where continuum states were corrected in agreement with Eq. (26). 
As seen from Fig. 6, the subtracting of the freestate contribution $N_{\text {free, } \varepsilon}$ from the continuum states reduces significantly the result for the excitation energy $E_{e x}$ (solid line) in the case of high enough temperatures. Note also that the correct description of continuum states can be important in the case of nuclei beyond the stability line where the Fermi energy is located close to the edge of potential well.

\section{Summary and conclusion}

In present paper, we have applied the standard thermodynamic derivation of the statistical level density $\rho\left(E_{e x}\right)$ and the statistical level density parameter $K$ to the finite nuclei. The key element of such kind of approach is the excitation energy $E_{e x}$ of a nucleus. In general, the calculations of the excitation energy $E_{e x}$ of strong interacting system like a nucleus is extremely complicate problem. A significant progress is here achieved by use of the Landau's conception of quasiparticles where the excitation energy $E_{e x}$ is derived similarly to a noninteracting Fermi-gas and depends thereby on the single-particle level density $g(\varepsilon)$.

Using the Wigner distribution function in phase space $f(\mathbf{r}, \mathbf{p})$, we have presented the semiclassical derivation of the single-particle level density $g(\varepsilon)$. Two approximations, namely Thomas - Fermi (TF) and Extended Thomas - Fermi (ETF), have been analyzed. Evaluating the number of particles $N_{\varepsilon}$ embedded in potential well $V(\mathbf{r})$ with energy below $\varepsilon$, we have shown that the $\hbar^{2}$-corrections to $N_{\varepsilon}$ caused by the ETF approximation play a minor role and we have restricted our consideration by the Thomas - Fermi approximation only.

Applying the Landau's conception of quasiparticles, we have introduced the nucleon effective mass $m^{*}<m$. It was shown that the effective mass $m^{*} \neq m$ affects significantly the slope of curve $g(\varepsilon)$ for the bound states with energy $e<0$ in vicinity of the Fermi energy $\varepsilon \approx \varepsilon_{F}$, see Fig. 3. This fact plies an important role for the calculations of the nuclear excitation energy $E_{e x}$ and the statistical level density parameter $K$. Considering the realistic finite-depth potentials $V(\mathbf{r})$, we have paid a special attention to the accuracy of derivation of the level density $g(\varepsilon)$ for the continuum states to prevent a spurious contribution to the excitation energy $E_{e x}$ which is caused by free space states and which is not related to the potential well $V(r)$. Our numerical calculations for the Woods - Saxon potential show that the correct subtracting of the free-state contribution from the continuum states reduces significantly the result for the excitation energy $E_{e x}$ in the case of high enough temperatures, see Fig. 6.

The numerical calculations of the inverse level density parameter $K$ have been performed for the Woods - Saxon potential well. We have used a standard parameterization of WS potential without the adjustable parameters. We have shown (see Fig. 5) that the Thomas - Fermi approximation with the effective mass of nucleon $m^{*}=0.7 m$ provides a quite satisfactory description of average $A$-dependency of the statistical level density parameter $K$.

\section{REFERENCES}

1. Ericson T. The statistical model and nuclear level densities // Adv. Phys. - 1960. - Vol. 9, No. 3. - P. 425 $-511$.

2. Bor O., Mottel'son B. The structure of the atomic nucleus. - Vol. 1. - Moskva: Mir, 1971. - 456 p. (Rus)

3. Gilbert A., Cameron A. A composite nuclear-level density formula with shell corrections // Can. J. Phys. - 1965. - Vol. 43, No. 8. - P. 1446 - 1496.

4. Huizenga J.R., Moretto L.G. Nuclear Level Densities // Ann. Rev. Nucl. Sci. - 1972. - Vol. 22. - P. 427 464.

5. Stavinskij V.S. The level density of nuclei // EchAYa. - 1972. - Vol. 3, No. 4. - P. 832 - 893. (Rus)

6. Kataria S.K., Ramamurthy V.S., Kapoor S.S. Semiempirical nuclear level density formula with shell effects // Phys. Rev. C. - 1978. - Vol. 18, No. 1. P. 549 - 563.

7. Ignatyuk A.V., Mikhailov I.N., Molina L.N. et al. The shape of the heated fast-rotating nuclei // Nucl. Phys.
A. - 1980. - Vol. 346, No. 1 - 2. - P. 191 - 215.

8. Shlomo S., Kolomietz V.M. Hot Nuclei // Rep. Prog. Phys. - 2005. - Vol. 68, No. 1. - P. 1 - 76.

9. Bogila Ye.A., Kolomietz V.M., Sanzhur A.I. Nuclear level density with fixed excitation number // Z. Phys. A. - 1992. - Vol. 341, No. 3. - P. 373 - 381.

10. Shlomo S., Bogila Ye.A., Kolomietz V.M., Sanzhur A.I. Fixed exciton number level density for a finite potential well // Z. Phys. A. - 1995. - Vol. 353, No. 1. - P. 27 - 30.

11. Lifshits E.M., Pitaevskij L.P. Physical kinetics. Moskva: Nauka, 1979. - 527 p. (Rus)

12. Migdal A.B. The theory of finite Fermi systems and properties of atomic nuclei. - Moskva: Nauka, 1965. 572 p. (Rus)

13. Kirzhnits D.A. Field methods in the theory of many particles. - Moskva: Atomizdat, 1963. - 343 p. (Rus)

14. Grammaticos B., Voros A. Semiclassical approximations for nuclear Hamiltonians. I. Spin-independent 
potentials // Ann. of Phys. - 1979. - Vol. 123, No. 2. P. 359 - 380.

15. Ring P., Schuck P. The Nuclear Many-Body Problem. - Berlin: Springer-Verlag. 1980. - 711 p.

16. Prakash M., Wambach J., Ma Z.Y. Effective mass in nuclei and the level density parameter // Phys. Lett. B. - 1983. - Vol. 128, No. 3 - 4. - P. 141 - 146.
17. Shlomo S., Kolomietz V.M., Dejbakhsh H. Single particle level density in finite depth potential well // Phys. Rev. C. - 1997. - Vol. 55, No. 4. - P. 1972 1981.

18. Levinson $N$. On the uniqueness of the potential in a Schrödinger equation for a given asymptotic phase // Danske Vid. Selsk., Mat-fys. Medd. - 1949. - Vol. 25, No. 9. - P. 129.

\title{
В. М. Коломієць
}

Інститут ядерних досліджень НАН Украӥни, Київ

\section{СТАТИСТИЧНА ГУСТИНА ЗБУДЖЕНИХ СТАНІВ ЯДЕР}

Квазікласичне наближення застосовано до розрахунку одночастинкової та статистичної густини збуджених станів ядер. Використано концепцію Ландау про ефективну масу нуклона $m^{*}<m$. Даний підхід забезпечує коректний опис внеску рівнів суцільного спектра в реалістичних ядерних потенціалах скінченої глибини. Показано, що стани суцільного спектра не впливають суттєво на термодинамічні розрахунки при достатньо малих температурах $T \leq 1 \mathrm{MeB}$, але значно змінюють результати при високих температурах. Використовуючи стандартний потенціал Вудса - Саксона та ефективну масу нуклонів $m^{*}=0,7 m$, обрахована залежність статистичного параметрf густини рівнів $K$ від числа нуклонів $A$, яка добре узгоджується із експериментальними даними.

Ключові слова: густина рівнів, збуджені ядра, ядерна температура, ефективна маса нуклона, суцільний спектр рівнів.

\section{В. М. Коломиец}

\author{
Институт ядерных исследований НАН Украины, Киев
}

\section{СТАТИСТИЧЕСКАЯ ПЛОТНОСТЬ ВОЗБУЖДЕННЫХ СОСТОЯНИЙ ЯДЕР}

Квазиклассическое приближение использовано для расчета одночастичной и статистической плотности возбужденных состояний ядер. Использована концепция Ландау эффективной массы нуклона $m^{*}<m$. Данный подход обеспечивает корректное описание вклада уровней сплошного спектра для реалистических ядерных потенциалов конечной глубины. Показано, что состояния сплошного спектра не влияют существенно на термодинамические расчеты при достаточно малых температурах $T \leq 1 \mathrm{MэB,} \mathrm{но} \mathrm{значительно} \mathrm{меняют}$ результаты при высоких температурах. С использованием стандартного потенциала Вудса-Саксона и эффективной массы нуклона $m^{*}=0,7 m$ вычислена зависимость статистического параметра плотности уровней $K$ от числа нуклонов $A$, которая находится в хорошем согласии с экспериментальными данными.

Ключевые слова: плотность уровней, возбужденные ядра, ядерная температура, эффективная масса нуклона, сплошной спектр уровней. 